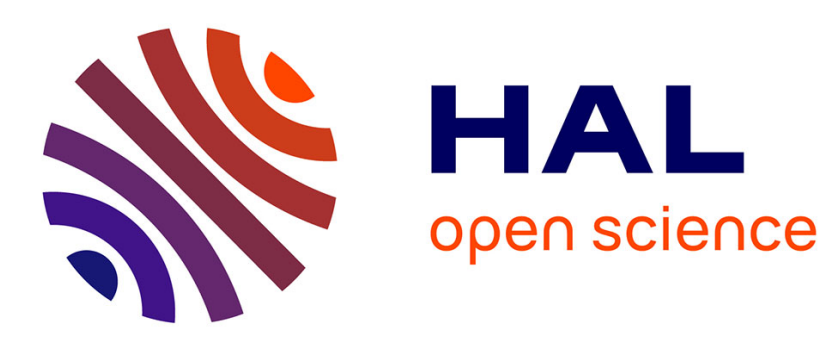

\title{
Record mondial d'éclairs dans le bassin du Congo
}

\author{
Serge Soula, Jean Kigotsi Kasereka, Jean Georgis, Christelle Barthe
}

\section{To cite this version:}

Serge Soula, Jean Kigotsi Kasereka, Jean Georgis, Christelle Barthe. Record mondial d'éclairs dans le bassin du Congo. La Météorologie, 2019, 104, pp.34. hal-02056985

\section{HAL Id: hal-02056985 \\ https://hal.univ-reunion.fr/hal-02056985}

Submitted on 2 Sep 2021

HAL is a multi-disciplinary open access archive for the deposit and dissemination of scientific research documents, whether they are published or not. The documents may come from teaching and research institutions in France or abroad, or from public or private research centers.
L'archive ouverte pluridisciplinaire HAL, est destinée au dépôt et à la diffusion de documents scientifiques de niveau recherche, publiés ou non, émanant des établissements d'enseignement et de recherche français ou étrangers, des laboratoires publics ou privés.

\section{(c)(1)}

Distributed under a Creative Commons Attribution| 4.0 International License 


\title{
Record mondial d'éclairs dans le bassin du Congo
}

\author{
Serge Soula ${ }^{1}$, Jean Kigotsi Kasereka ${ }^{1,2}$, Jean-François Georgis ${ }^{1}$, \\ Christelle Barthe ${ }^{3}$ \\ 1. Laboratoire d'aérologie, Université de Toulouse / CNRS, Toulouse, France \\ 2. Département de physique, Faculté des sciences, Université de Kinshasa, \\ République démocratique du Congo \\ 3. Laboratoire de l'atmosphère et des cyclones, Université de La Réunion / \\ Météo-France / CNRS, Saint-Denis, France
}

serge.soula@aero.obs-mip.fr

\section{Résumé}

La climatologie des éclairs dans le bassin du Congo est analysée à l'aide des données du réseau World Wide Lightning Location Network (WWLLN) de 2005 à 2013. Une comparaison avec les données du capteur optique spatial LIS montre que l'efficacité de détection relative du réseau WWLLN passe d'environ $1,7 \%$ au début de la période à $5,9 \%$ en 2013. Ces valeurs moyennes sur l'ensemble de la zone sont faibles, mais concernent la totalité des éclairs (intranuage et nuage-sol) et permettent d'avoir une bonne représentativité de l'activité orageuse particulièrement forte dans la zone d'étude. Le taux d'éclair moyen décrit un cycle annuel avec de fortes proportions mensuelles d'octobre à mars de l'ordre de 9 à $11 \%$ et des valeurs au moins deux fois plus faibles de juin à août. La distribution a climatologie globale des éclairs est connue depuis le développement des observations optiques spatiales, notamment grâce au détecteur OTD (Optical Transient Detector) installé à bord du satellite MicroLab-1 pour une mission de mai 1995 à mars 2000 (Christian et al., 2003), puis au capteur LIS (Lightning Imaging Sensor) sur la mission TRMM (Tropical Rainfall Measuring Mission) de 1997 à 2014 dans la zone située entre $38^{\circ} \mathrm{S}$ et $38^{\circ} \mathrm{N}$ (Albrecht et al., 2016). La figure 1 constitue une synthèse des résultats de ces observations en représentant le taux de densité d'éclairs FRD (Flash Rate Density, utilisé par la suite) avec son unité spécifique en éclairs $\mathrm{km}^{-2} \mathrm{an}^{-1}$. Les valeurs obtenues sont très variables d'une région à l'autre et vont donc dépendre de la résolution spatiale utilisée pour le calcul de ce taux de densité. De même, les valeurs maximales peuvent être différemment localisées en changeant cette résolution. Ainsi, avec une résolution de $0,1^{\circ} \times 0,1^{\circ}$, le maximum est trouvé dans la région du lac Maracaibo, dans l'État de Zulia au Venezuela, avec une valeur d'environ 230 éclairs $\mathrm{km}^{-2} \mathrm{an}^{-1}$
(Albrecht et al., 2016). Toutefois, ce maximum étant très localisé, avec une résolution plus basse $\left(0,25^{\circ} \times 0,25^{\circ}\right)$, le maximum était trouvé dans une grande région de la République démocratique du Congo (RDC), avec des valeurs de plus de 80 éclairs $\mathrm{km}^{-2} \mathrm{an}^{-1}$ localement et plus de 30 éclairs $\mathrm{km}^{-2} \mathrm{an}^{-1}$ sur 3 millions de $\mathrm{km}^{2}$ (Christian et al., 2003). Avec une résolution de $0,1^{\circ} \times 0,1^{\circ}$, Cecil et al. (2014) ont trouvé le plus fort taux en Afrique centrale, avec un pic de 160 éclairs $\mathrm{km}^{-2} \mathrm{an}^{-1}$ dans l'est de la RDC. La figure 1 donne une idée de la répartition mondiale du FRD à haute résolution. Albrecht et al. (2016) ont classé les zones de fort FRD (hot spot) calculé à une résolution de $0,1^{\circ} \times 0,1^{\circ}$ en considérant une distance minimum de $100 \mathrm{~km}$ entre ces zones. Si la première zone se trouve bien au Venezuela, on en trouve six parmi les dix premières (classées en 2, 3, 5, 8, 9 et 10) localisées sur le territoire de la RDC avec des taux de densité allant de 205 à 127 éclairs $\mathrm{km}^{-2} \mathrm{an}^{-1}$. On voit donc bien la concentration de ces régions à forte activité d'éclairs dans le bassin du Congo. L'analyse détaillée de la climatologie de l'activité d'éclairs

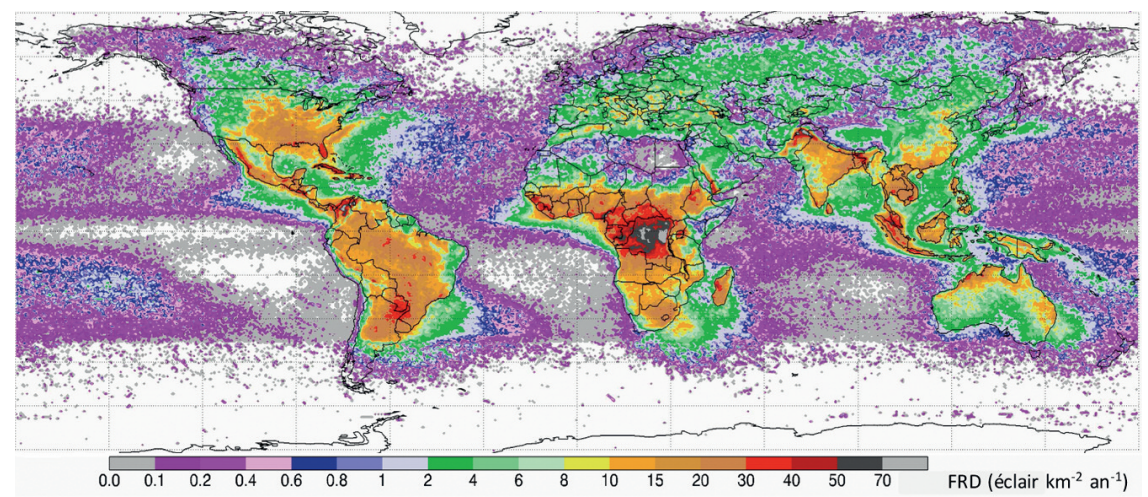

Figure 1. Climatologie mondiale de l'activité d'éclairs issue des données des capteurs spatiaux OTD et LIS à haute résolution. L'échelle de couleur représente les valeurs du taux de densité d'éclairs annuel en éclairs $\mathrm{km}^{-2} \mathrm{an}^{-1}$. (C) Nasa. 
méridienne des éclairs montre un renforcement entre $5^{\circ} \mathrm{S}$ et $5^{\circ} \mathrm{N}$ avec un maximum entre $1^{\circ} \mathrm{S}$ et $2^{\circ} \mathrm{S}$. La répartition géographique montre un maximum très marqué dans l'est de la République démocratique du Congo (RDC), quelles que soient l'année de référence et la période de l'année. En 2013, la densité annuelle d'éclairs estimée en tenant compte de l'efficacité de détection et du nombre de jours d'orages, calculés avec une résolution de $0,1^{\circ} \times 0,1^{\circ}, \mathrm{y}$ atteignent respectivement 218 éclairs $\mathrm{km}^{-2} \mathrm{an}^{-1}$ et 189 jours. Le nombre maximum d'éclairs par jour d'orage se situe dans la même région, ce qui signifie que les orages $y$ sont, soit plus nombreux, soit plus intenses, soit plus stationnaires, soit plusieurs des trois caractéristiques à la fois. Cette région se situe à l'ouest de la chaîne de montagnes des Virunga qui présente une altitude supérieure à $\mathbf{3 0 0 0}$ mètres et qui joue un rôle dans l'initialisation des orages au cours de l'année. De plus, la présence du courant jet africain oriental de basse altitude produit l'instabilité nécessaire au développement local de systèmes convectifs de moyenne échelle (MCS) à l'origine de cette forte activité. Ces MCS peuvent ensuite se propager ou se régénérer vers l'ouest sur une distance liée à l'influence de la phase des ondes de Kelvin se propageant vers l'est. proposée dans cet article vise à améliorer la compréhension du développement des orages dans la région du bassin du Congo, en termes de localisation, de répartition annuelle, de relations avec les caractéristiques de l'environnement, de conditions de formation, de durée de vie et de déplacement.

\section{Ce que détecte le réseau WWLLN}

Le réseau WWLLN (World Wide Lightning Location Network, www.wwlln.net) est un réseau mondial de détection des éclairs intranuage et nuage-sol basé sur la détection des rayonnements électromagnétiques qu'ils émettent à très basse fréquence (TBF). Les sources de ces rayonnements sont localisées à l'aide de la technique dite des différences de temps d'arrivée d'un même signal sur plusieurs stations du réseau (Dowden et al., 2002). Depuis son implantation en mars 2003, le réseau WWLLN a été amélioré en termes de nombre de stations et de développement de l'algorithme de traitement (Rodger et al., 2008) et son efficacité de détection a été estimée par plusieurs auteurs en comparaison avec les données satellitaires (Bovalo et al., 2012 ; Soula et al., 2016) ou avec celles d'autres réseaux de détection au sol (Abarca et al., 2010). Il a été noté que des capteurs supplémentaires de 2003 à 2007 amélioraient considérablement l'efficacité de détection (Rodger et al., 2008) : de 10,6 millions d'éclairs en mars-décembre 2003 avec 11 capteurs, à 28,1 millions pour la même période en 2007 avec 30 capteurs. En outre, ils ont noté que les améliorations apportées à l'algorithme utilisé pour reconstituer les éclairs à partir des détections du réseau WWLLN ont augmenté l'efficacité de détection de $63 \%$ lorsqu'il était appliqué aux données de 2007. En 2014, le réseau WWLLN comptait plus de 60 capteurs répartis sur la planète, comme l'indique la figure 2 . Même s'il est capable de détecter à la fois les éclairs nuage-sol et intranuage, étant donné que les pics de courant sont beaucoup plus forts pour les éclairs nuage-sol, l'efficacité de détection est environ deux fois plus grande pour ces éclairs. Elle dépend également de la région considérée, car la couverture du réseau n'est pas homogène. Ainsi, Bovalo et al. (2012) trouvait une efficacité de détection à 8,5\% en 2011 par rapport à la totalité des éclairs détectés par le capteur LIS pour le bassin ouest de l'océan Indien. Dans le territoire du Bassin du Congo, Soula et al. (2016) ont estimé l'efficacité de détection par rapport aux données du capteur LIS à près de $6 \%$ pour l'année 2013. Abarca et al. (2010) ont estimé

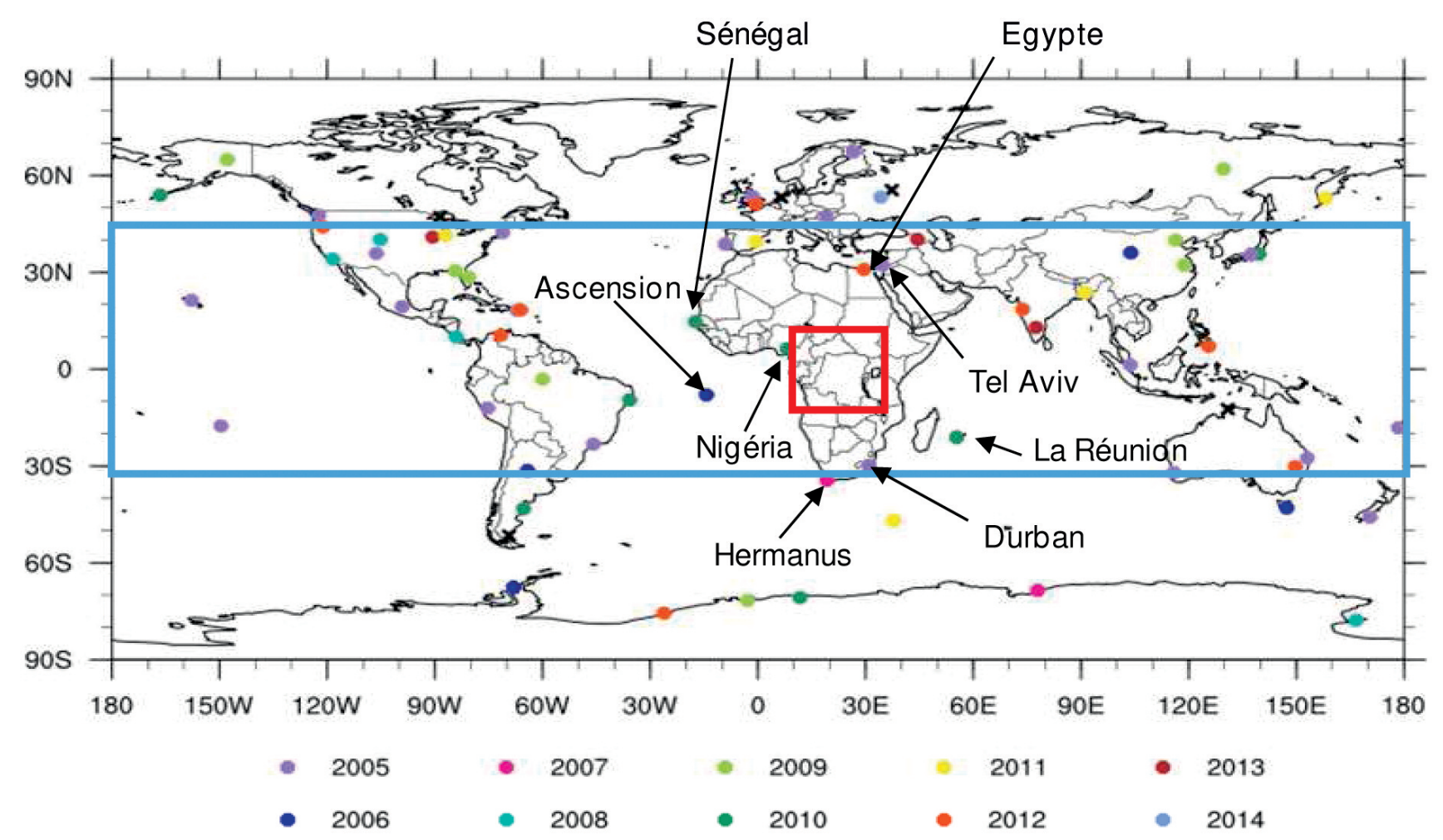

Figure 2. Carte des stations du réseau WWLLN. La couleur correspond à l'année d'installation. Le carré rouge et le grand rectangle bleu indiquent respectivement la zone d'étude et la couverture du capteur spatial LIS. Tiré de Soula et al. (2016). 


\section{Abstract}

\section{World record of lightning in the Congo Basin}

Lightning climatology in the Congo Basin is analyzed using data from the World Wide Lightning Location Network (WWLLN) from 2005 to 2013. A comparison with data from the optical space sensor LIS shows that the relative detection efficiency of the WWLLN increased from approximately $1.7 \%$ at the beginning of the period to $5.9 \%$ in 2013 . These mean values over the entire area are low but related to all lightning flashes (intracloud and cloud-to-ground) and allow, however, obtaining a good representation of thunderstorm activity particularly strong in the study area. The average flash rate describes an annual cycle with high monthly proportions from October to March from 9 to $11 \%$, and values at least 2 times lower from June to August. The meridian distribution of lightning shows a reinforcement between $5^{\circ} \mathrm{S}$ and $5^{\circ} \mathrm{N}$ with a maximum between $1^{\circ} \mathrm{S}$ and $2^{\circ} \mathrm{S}$. The geographical distribution shows a very strong maximum in the East of the Democratic Republic of Congo (DRC), whatever the reference year and the period of the year. In 2013 , the estimated annual lightning density taking into account DE and the number of thunderstorm days, calculated with a resolution of $0.1^{\circ} \times$ $0.1^{\circ}$, reached 218 flashes $\mathrm{km}^{-2}$ year $^{-1}$ and 189 days, respectively. The maximum number of flashes per thunderstorm is in the same area, which means that thunderstorms are there, either more numerous, or more intense, or more stationary, or several of the three characteristics. This region is located west of the Virunga mountain range at an altitude that reaches 3000 meters and plays a role in the initiation of thunderstorms during the year. In addition, the presence of the South African Easterly Jet (AEJ-S) at low altitude produces the instability necessary to the local development of Mesoscale Convective Systems (MCS) at the origin of this strong activity. These MCS can then propagate or regenerate to the west over a distance related to the influence of the phase of Kelvin waves propagating eastward. l'efficacité de détection par rapport aux éclairs nuage-sol sur le territoire américain et trouvent autour de $10 \%$ pour l'état du réseau dans la période 2008-2009. Ils trouvent aussi que l'efficacité de détection est fortement dépendante des pics de courant des arcs-en-retour des éclairs nuage-sol. En effet, les capteurs du réseau WWLLN détectent les arcs d'éclairs nuage-sol ou des signaux produits par des éclairs intranuage, et il est donc nécessaire de reconstruire les éclairs. En testant plusieurs critères de temps et d'espace pour les arcs associés dans un même éclair, nous avons adopté les valeurs de $0,5 \mathrm{~s}$ et $20 \mathrm{~km}$, respectivement. Les données du réseau WWLLN enregistrées pendant neuf ans (2005 à 2013) ont été utilisées pour étudier l'évolution temporelle de l'activité d'éclairs et sa répartition géographique sur une vaste zone matérialisée sur la figure 2 par le carré rouge $\left(15^{\circ} \mathrm{S}-10^{\circ} \mathrm{N}\right.$ en latitude et $10^{\circ} \mathrm{E}-35^{\circ} \mathrm{E}$ en longitude) qui inclut et entoure le territoire de la RDC (Soula et al., 2016).

\section{Les données du réseau WWLLN sur le bassin du Congo de 2005 à 2013}

Le tableau 1 présente plusieurs paramètres liés à l'activité annuelle d'éclairs dans la zone de l'étude. La première et la deuxième colonne indiquent le nombre d'éclairs détectés par le réseau WWLLN $\left(N_{\mathrm{W}}\right)$ et par le capteur LIS $\left(N_{\mathrm{L}}\right)$, respectivement, qui sont également représentés sur la figure 3 afin de visualiser leur évolution de 2005 à 2013. Après une première période de quatre ans où $N_{\mathrm{W}}$ est à peu près

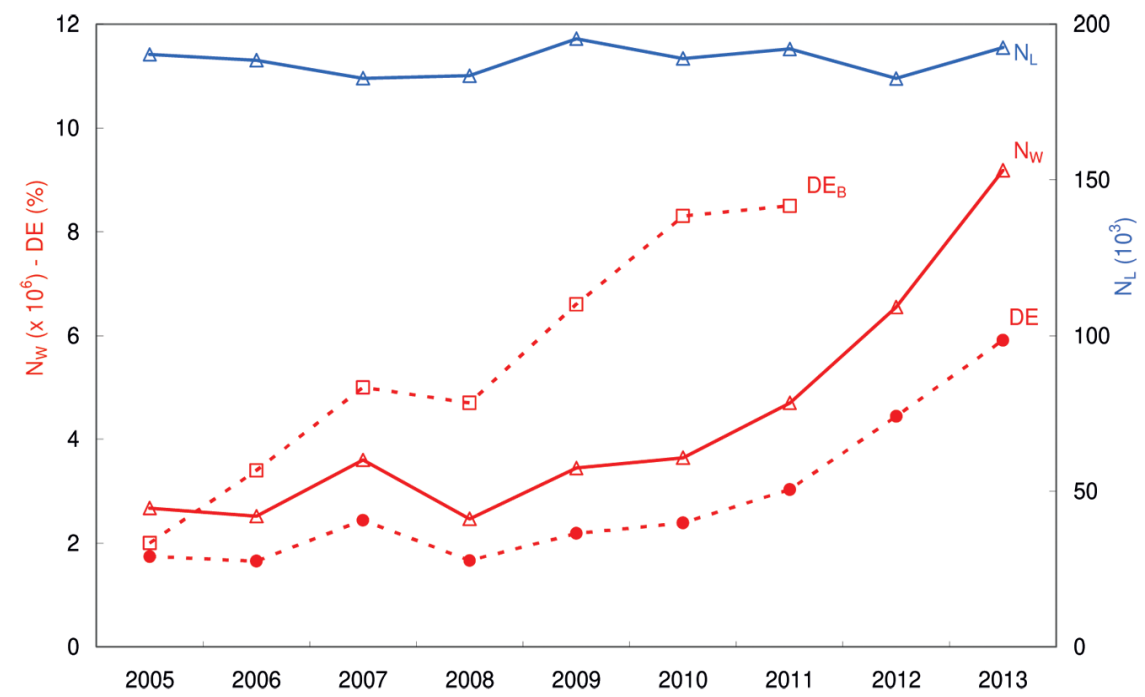

Figure 3. Nombre d'éclairs détectés par le réseau WWLLN $\left(N_{W}\right)$ et par le capteur LIS $\left(N_{1}\right)$, efficacité de détection DE pour WWLLN par rapport à LIS et $\mathrm{DE}_{\mathrm{B}}$ pour WWLLN dans le bassin ouest-océanique indien, d'après Bovalo et al. (2012). Adapté de Soula et al. (2016).

Tableau 1. Nombre d'éclairs détectés par le réseau WWLLN $\left(N_{W}\right)$ et le capteur LIS $\left(N_{L}\right)$, nombre total d'éclairs estimé $\left(N_{0}\right)$. Efficacité de détection du réseau WWLLN (DE) par rapport à celle du capteur LIS et $\left(\mathrm{DE}_{\mathrm{B}}\right.$ ) estimée par Bovalo et al. (2012) pour le bassin ouest-océanique indien. Nombre maximum de jours d'orage $\left(S D_{\max }\right)$, densité maximale de taux d'éclairs, issue du réseau WWLLN $\left(F R D_{\max }\right)$ et estimée en tenant compte de $\mathrm{DE}\left(\mathrm{FRD}_{\max }^{\prime}\right)$. Multiplicité moyenne $\left(M_{\mathrm{m}}\right)$ et proportion d'éclairs ayant produit un seul arc-en-retour $(M=1)$

\begin{tabular}{ccccccccccc} 
& $N_{\mathrm{W}}$ & $N_{\mathrm{L}}$ & $N_{0}$ & $\begin{array}{c}\mathrm{DE} \\
(\%)\end{array}$ & $\begin{array}{c}\mathrm{DEB} \\
(\%)\end{array}$ & $\begin{array}{c}\mathrm{SD}_{\max } \\
(\text { jour })\end{array}$ & $\begin{array}{c}\mathrm{FRD}_{\max } \\
\left(\mathrm{km}^{-2} \mathrm{an}^{-1}\right)\end{array}$ & $\begin{array}{c}\mathrm{FRD}_{\text {max }} \\
\left(\mathrm{km}^{-2} \mathrm{an}^{-1}\right)\end{array}$ & $\begin{array}{c}M_{\mathrm{m}} \\
\left(\text { éclair-1) }^{-1}\right.\end{array}$ & $\begin{array}{c}M=1 \\
(\%)\end{array}$ \\
\hline 2005 & 2676276 & 190308 & 153761877 & 1,7 & 2,0 & 114 & 2,7 & 155,2 & 1,08 & 93,7 \\
\hline 2006 & 2516580 & 188449 & 152259873 & 1,6 & 3,4 & 154 & 1,9 & 115,1 & 1,13 & 89,0 \\
\hline 2007 & 3602064 & 182653 & 147568838 & 2,4 & 5,0 & 131 & 2,7 & 109,0 & 1,11 & 90,6 \\
\hline 2008 & 2467176 & 153466 & 148233792 & 1,7 & 4,7 & 130 & 1,9 & 115,1 & 1,09 & 91,9 \\
\hline 2009 & 3446317 & 195316 & 157808157 & 2,2 & 6,6 & 143 & 3,9 & 180,3 & 1,12 & 90,3 \\
\hline 2010 & 3643387 & 188984 & 152692134 & 2,4 & 8,3 & 157 & 4,4 & 184,5 & 1,14 & 88,4 \\
\hline 2011 & 4701732 & 192007 & 155134607 & 3,0 & 8,5 & 167 & 4,6 & 152,8 & 1,18 & 85,4 \\
\hline 2012 & 6550235 & 182560 & 147501778 & 4,4 & - & 167 & 8,2 & 185,1 & 1,22 & 83,0 \\
\hline 2013 & 9181456 & 192443 & 155486879 & 5,9 & - & 189 & 12,9 & 218,0 & 1,28 & 79,62 \\
\hline
\end{tabular}


constant, sauf en 2007, il augmente de manière significative et atteint en 2013 environ 3,5 fois la valeur de 2005. Au contraire, $N_{\mathrm{L}}$ varie peu au cours de la même période, entre 182560 en 2012 et 195316 en 2009. L'efficacité de détection du capteur LIS n'ayant pas changé au cours de la période, nous pouvons estimer que l'activité n'a pas changé de manière significative et donc l'augmentation du nombre d'éclairs détectés par le réseau WWLLN est due à une amélioration de l'efficacité de détection.

Le tableau 1 montre également la multiplicité moyenne annuelle $M_{\mathrm{m}}$ et la proportion d'éclairs avec une multiplicité égale à $1(M=1)$, la multiplicité étant le nombre d'arcs dans un même éclair. Tout d'abord, on peut noter que la multiplicité est faible, entre 1,08 en 2005 et 1,28 en 2013, ce qui est nettement inférieur à la multiplicité généralement constatée pour les réseaux régionaux puisqu'il est proche de 2 pour les éclairs nuage-sol négatifs et un peu supérieur à 1 pour les positifs, comme aux États-Unis avec le réseau NLDN (National Lightning Detection Network) (Orville et Huffines, 2001). Deuxièmement, la multiplicité moyenne augmente lorsque davantage d'éclairs sont détectés, c'est-à-dire lorsque DE augmente, tandis que la proportion d'éclairs avec $M=1$ diminue évidemment. Ainsi, l'augmentation de DE conduit à détecter plus d'arcs en retour, ce qui augmente à la fois le nombre d'éclairs et la multiplicité des éclairs détectés.

La figure 4 présente la proportion mensuelle moyenne d'éclairs détectés par le réseau WWLLN sur toute la zone d'étude, ainsi que les valeurs extrêmes de cette proportion dans un intervalle de confiance de $95 \%$. L'écart-type autour de la valeur moyenne varie de 12 à $25 \%$ en valeur relative. Pour chaque année, le même cycle est observé : une proportion mensuelle d'éclairs minimum autour de $4 \%$ pendant les mois de juin, juillet et août ; une proportion mensuelle maximum centrée autour des mois de décembre et janvier de durée variable selon l'année et de l'ordre de 9 à $11 \%$ en moyenne.

Les valeurs annuelles de l'efficacité de détection $\mathrm{DE}$ sont indiquées dans le tableau 1 et comparées à celles de $\mathrm{DE}_{\mathrm{B}}$ trouvée par Bovalo et al. (2012) pour le sud-ouest de l'océan Indien (SWIO). $\mathrm{DE}$ augmente continuellement après 2008, passant de $1,7 \%$ en 2008 à 5,9\% en 2013. Cette augmentation est en

\section{Estimation de l'efficacité de détection}

L'efficacité de détection pour un système de détection d'éclairs est relative : elle peut être calculée par rapport à un autre système qui est censé détecter la presque totalité des événements. Pour le territoire de l'étude et sur sa période, les données du capteur LIS vont être utilisées, mais il faut tenir compte du fait que ce capteur était sur un satellite défilant. En effet, le capteur LIS couvre un point de la surface terrestre pendant 90 secondes au cours de son passage, mais il a un DE d'environ $90 \%$, tandis que le réseau WWLLN réalise une couverture continue, mais avec un $D E$ très faible. Une première étape consiste à estimer ce que verrait le capteur LIS avec une observation continue sur le territoire à l'échelle d'une année.

Tout d'abord, nous calculons la surface $S_{A}$ couverte parle capteur LIS sur l'ensemble de ses orbites entre $38^{\circ} \mathrm{S}$ et $38^{\circ} \mathrm{N}$ (cadre bleu sur la figure 2). Dans cette bande, l'élément de surface $\mathrm{d} S$ est :

$$
\mathrm{d} S=R_{\mathrm{T}} \mathrm{d} \lambda R_{\mathrm{T}} \cos \lambda \mathrm{d} \varphi
$$

où $\lambda$ est la latitude, $\varphi$ la longitude et $R_{\mathrm{T}}$ le rayon de la Terre au niveau de l'équateur $\left(R_{\mathrm{T}}=6378 \mathrm{~km}\right)$.

En intégrant, on obtient :

$$
\begin{gathered}
S_{\mathrm{A}}=R_{\mathrm{T}}^{2} \iint \cos \lambda \mathrm{d} \lambda \mathrm{d} \varphi=R_{\mathrm{T}}^{2} \int_{0}^{2 \pi}\left|\int_{-38^{\circ}}^{38^{\circ}} \cos \lambda \mathrm{d} \lambda\right| \mathrm{d} \varphi \\
S_{\mathrm{A}}=4 \pi R_{\mathrm{T}}^{2} \times 0,6157
\end{gathered}
$$

Pendant une année de 366 jours (31 $622400 \mathrm{~s}$ ), le satellite TRMM couvre 5704 orbites. Ainsi, la durée moyenne d'une orbite est de 31622400 / $5704=$ 5543,9 secondes.

Pendant une orbite, la surface couverte au sol est $S_{0}=L \times 600 \mathrm{~km}$.

$L$ est la projection d'une orbite au sol :

$$
L=2 \pi R_{\mathrm{T}}=2 \pi \times 6378=40074 \mathrm{~km} .
$$

$600 \mathrm{~km}$ est la fauchée du capteur.

$\mathrm{S}_{0}=40074 \times 600=24044400 \mathrm{~km}^{2}$.
Ensuite, nous définissons un coefficient de surface :

$$
\alpha_{S}=\frac{S_{0}}{S_{\mathrm{A}}}=\frac{24044400}{4 \pi R_{\mathrm{T}}^{2} \times 0,6157}=0,07639
$$

Pendant une orbite, c'est-à-dire 5 543,9 secondes, la surface couverte est donc de $7,64 \%$ de la surface $S_{A}$. De plus, le satellite couvre chaque point de la surface pendant 90 secondes sur la durée de l'orbite. Un coefficient $\alpha_{t}$ est considéré pour la proportion du temps de l'orbite où un point est vu par le capteur LIS :

$$
\alpha_{t}=\frac{90}{5544}=0,0162
$$

Ainsi, le capteur LIS voit chaque point de la surface $S_{\mathrm{A}}$ couverte pendant $1,62 \%$ de la durée de l'orbite. Pour résumer, pendant une orbite, le capteur observe $7,64 \%\left(\alpha_{s}\right)$ de toute la surface couverte pendant ses passages successifs et chaque point est vu pendant $1,62 \%\left(\alpha_{t}\right)$ du temps de l'orbite. Par conséquent, le produit $\alpha_{s} \times \alpha_{t}$ représente la proportion du temps où un point de la bande $S_{\mathrm{A}}$ est vu par le capteur LIS sur une longue durée, par exemple sur une année :

$$
\begin{gathered}
\alpha=\alpha_{s} \times \alpha_{t}=0,0764 \times 0,0162= \\
0,00124 \text {, c'est-à-dire } 0,124 \%
\end{gathered}
$$

Ce coefficient est applicable pour tout point de $S_{\mathrm{A}}$ et donc pour tout point de la zone d'étude. En effet, nous pouvons considérer que statistiquement, étant donné le nombre d'orbites réalisées au cours d'une année, l'échantillonnage est représentatif de toute l'activité d'éclairs. Nous appliquons ce coefficient $\alpha$ au nombre $N_{\mathrm{L}}$ d'éclairs détectés par le capteur LIS pour estimer le nombre total d'éclairs $N_{0}$ produits dans la zone d'étude :

$$
N_{\mathrm{L}}=\alpha N_{0} \text { ou encore } N_{0}=N_{\mathrm{L}} / \alpha \text {. }
$$

Afin d'évaluer l'efficacité de détection pour le réseau WWLLN par rapport au capteur LIS, nombre d'éclairs détectés par $N_{0}$ :

$$
\mathrm{DE}=\frac{N_{\mathrm{W}}}{N_{0}}=\frac{N_{\mathrm{W}}}{N_{\mathrm{L}}} 0,00124
$$
nous prenons en compte $\alpha$ en divisant $N_{W}$ le

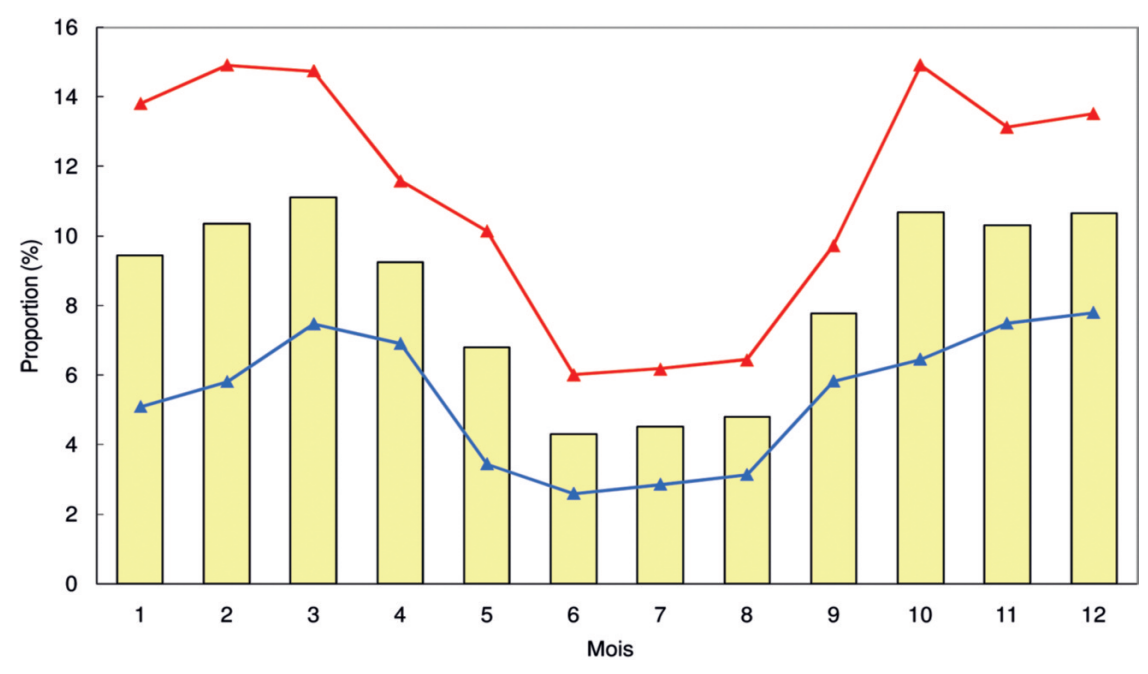

Figure 4. Proportion moyenne mensuelle d’éclairs détectés par le réseau WWLLN (\%) et intervalle de confiance à $95 \%$ (courbes rouge et bleue). Adapté de Soula et al. (2016). 
accord avec Bovalo et al. (2012) qui montre que $\mathrm{DE}_{\mathrm{B}}$ augmentait également de manière continue, mais plus rapidement, de 4,7\% en 2008 à 8,5\% en 2011. Une augmentation en 2007 est observée pour les deux, mais elle est plus prononcée pour DE. Bien entendu, l'augmentation globale de DE pour le réseau WWLLN par rapport au capteur LIS peut s'expliquer par une évolution du réseau. Une analyse détaillée de la répartition géographique de DE montre qu'elle est différente et qu'elle évolue différemment selon la localisation dans la zone d'étude, comme le montre la figure 5. En effet, on voit des valeurs de DE qui varient du simple au double dans la zone et une cohérence dans ces différences d'année en année. Les plus fortes valeurs de DE se trouvent notamment dans le sud de la zone avec des régions à plus de $10 \%$ en 2013.

\section{Répartition géographique de l'activité d'éclairs}

FRD est représenté sur la figure 6 pour chaque année avec une résolution de $0,1^{\circ}$. Dans chaque graphe, l'échelle est adaptée à la valeur maximale annuelle. La même forme de répartition est observée chaque année, avec notamment un maximum très marqué dans l'est de la $\mathrm{RDC}$, autour de $28^{\circ} \mathrm{E}$ de longitude et $1^{\circ}-2^{\circ} \mathrm{S}$ de latitude. Le contraste entre ce maximum et le reste de la zone d'étude est plus fort dans les dernières années de la période. En effet, des fortes valeurs de FRD sont visibles dans le centre de la RDC de 2005 à 2008, mais toutefois inférieures aux valeurs maximales dans l'est de la RDC. Le tableau 1 montre la valeur maximale $\mathrm{FRD}_{\max }$ pour chaque année, de 2005 à 2013. Tout d'abord, FRD $_{\max }$ oscille de 1,9 à 2,7 éclairs $\mathrm{km}^{-2} \mathrm{an}^{-1}$ dans la période 2005-2008, puis augmente fortement de 2008 à 2013. La valeur maximale est trouvée en 2013 avec 12,9 éclairs $\mathrm{km}^{-2} \mathrm{an}^{-1}$. Évidemment, FRD ${ }_{\text {max }}$ décrit la même évolution que $\mathrm{DE}$ et $N_{\mathrm{W}}$. Ainsi, $\mathrm{FRD}_{\text {max }}$ augmente d'un facteur 6 environ sur la période, tandis que $N_{\mathrm{W}}$ était seulement multiplié par un peu moins de 4 . La valeur de $\mathrm{FRD}_{\max }$ doit être considérée comme une valeur locale dans la zone d'étude. Si on estime cette densité en tenant compte de l'efficacité de détection, on trouve :

$$
\mathrm{FRD}_{\text {max }}^{\prime}=\frac{\mathrm{FRD}_{\text {max }}}{\mathrm{DE}} \times 100
$$

Les valeurs de FRD' ${ }_{\text {max }}$ sont reportées pour chaque année dans le tableau 1 et elles vont de 109,0 à
218,0 éclairs $\mathrm{km}^{-2} \mathrm{an}^{-1}$ à cette résolution de $0,1^{\circ}$. Bien entendu, ces grandes valeurs correspondent à l'activité totale d'éclairs, comme le verrait le capteur LIS avec une couverture complète et continue.

La figure 7 montre la distribution de FRD à partir des données du réseau WWLLN et moyennée sur deux périodes distinctes, 2005-2008 (figure 7a) et 2009-2013 (figure 7b). Cette figure confirme que le contraste entre le maximum dans l'est de la RDC et le reste de la région est beaucoup plus fort pendant la deuxième période. Ainsi, une large région du bassin central du Congo est aussi concernée par des valeurs élevées de FRD au cours de la première période. Les valeurs de FRD varient de 1,5 à 6,5 éclairs $\mathrm{km}^{-2} \mathrm{an}^{-1}$ dans le maximum principal, tandis qu'il varie de 1 à 2 éclairs $\mathrm{km}^{-2} \mathrm{an}^{-1}$ dans le bassin du Congo. Si on représente de la même manière l'activité d'éclairs observée par le capteur LIS (figure $7 \mathrm{c}-\mathrm{d}$ ) pour les mêmes périodes 2005-2008 et 20092013 , on ne voit plus de différence entre les deux périodes, car les deux distributions sont très proches en termes de valeurs et de contraste. Cela montre que la différence observée à partir des données du réseau WWLLN pour les deux périodes est due à une évolution différentielle de l'efficacité de détection dans la région étudiée.
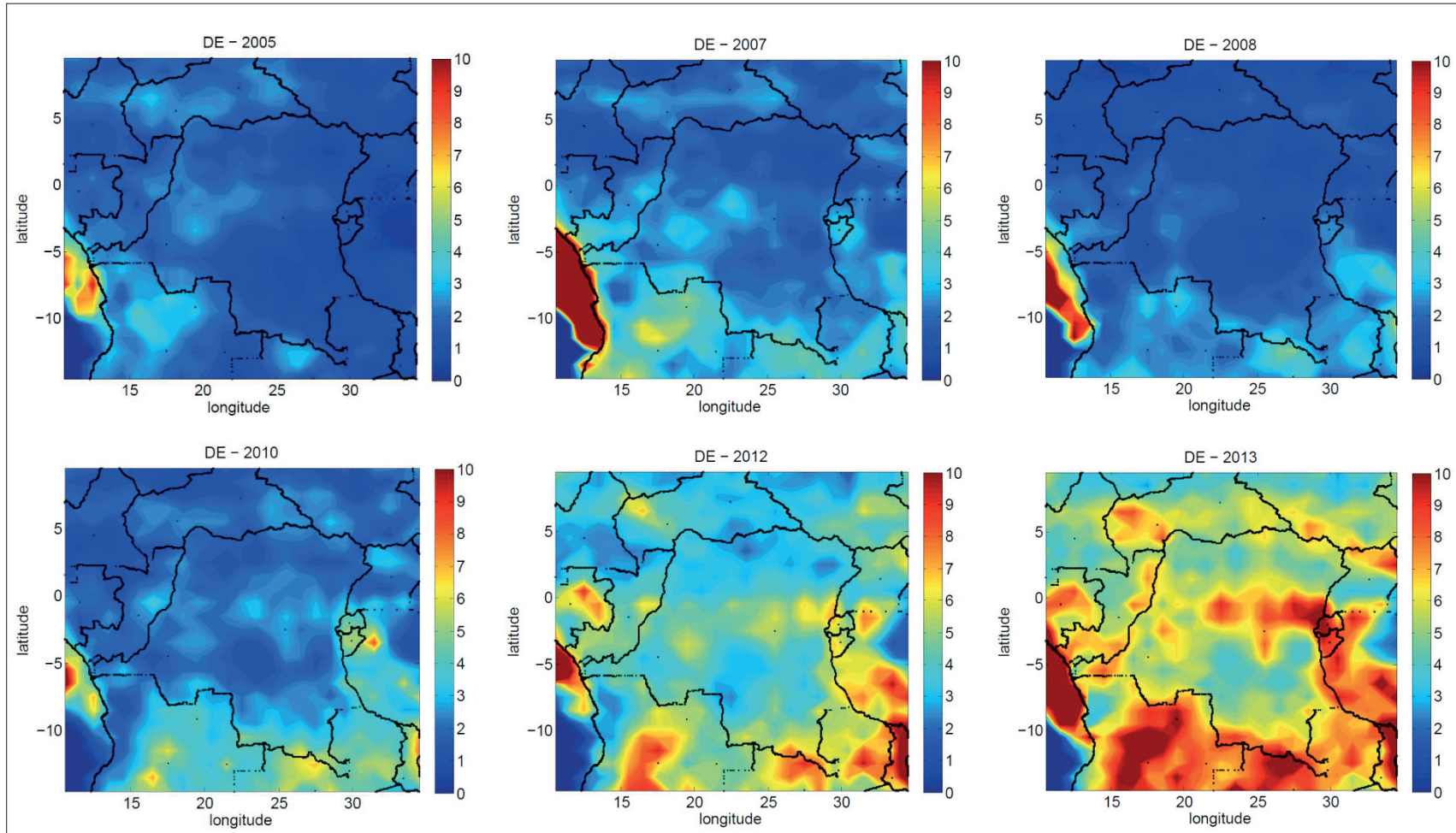

Figure 5. Distribution spatiale de l'efficacité de détection (DE) calculée à une résolution de $1^{\circ} \times 1^{\circ}$ dans la zone d'étude et pour plusieurs années. Tiré de Kigotsi (2018). 


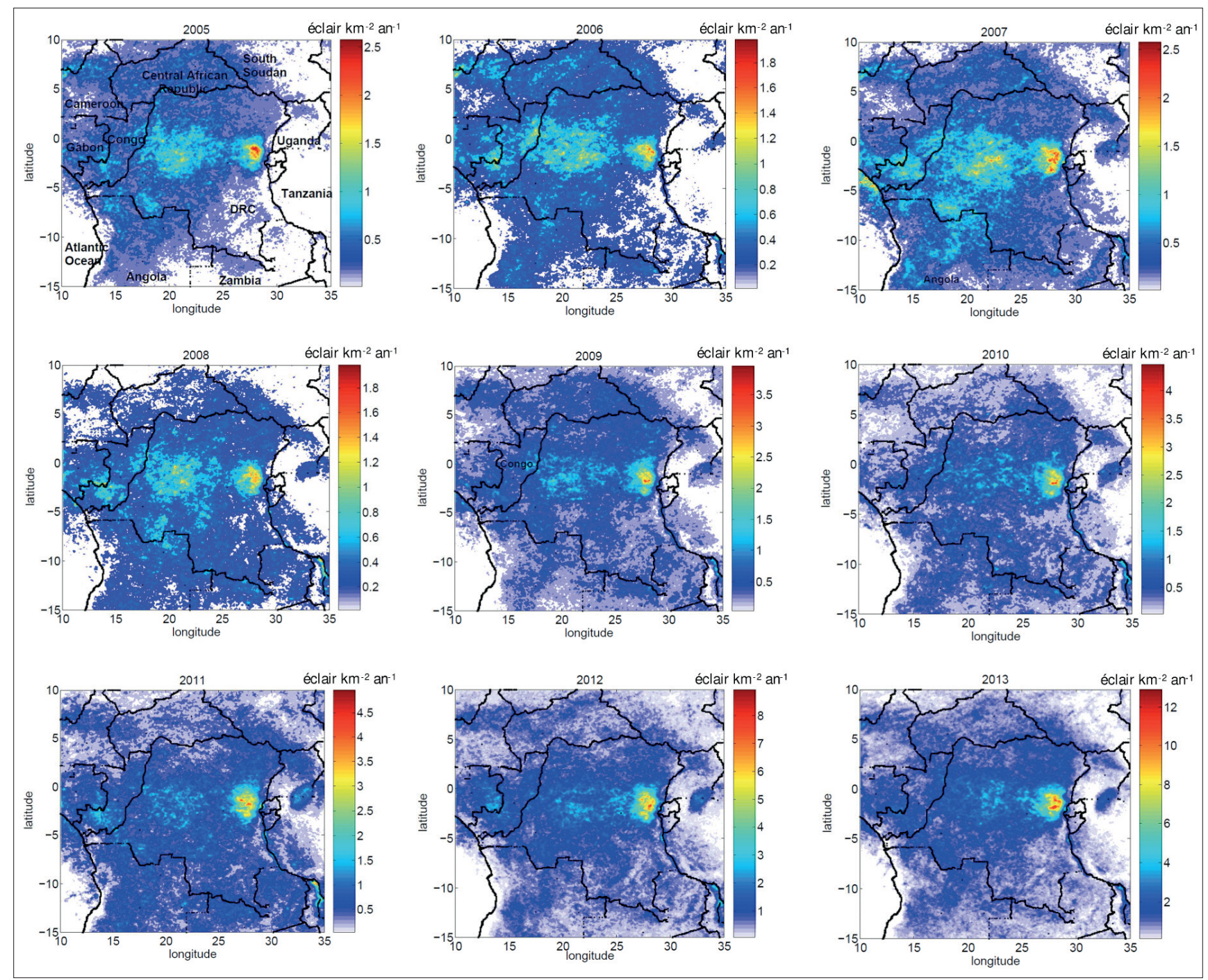

Figure 6. FRD (éclairs $\left.\mathrm{km}^{-2} \mathrm{an}^{-1}\right)$ vu par le réseau WWLLN dans la zone d'étude $\left(10^{\circ} \mathrm{E}-35^{\circ} \mathrm{E} ; 15^{\circ} \mathrm{S}-10^{\circ} \mathrm{N}\right)$ pour les années 2005 à 2013 . La résolution spatiale est de $0,1^{\circ} \times 0,1^{\circ}$ et l'échelle de couleur est adaptée aux valeurs maximales dans chaque graphe. Tiré de Soula et al. (2016).

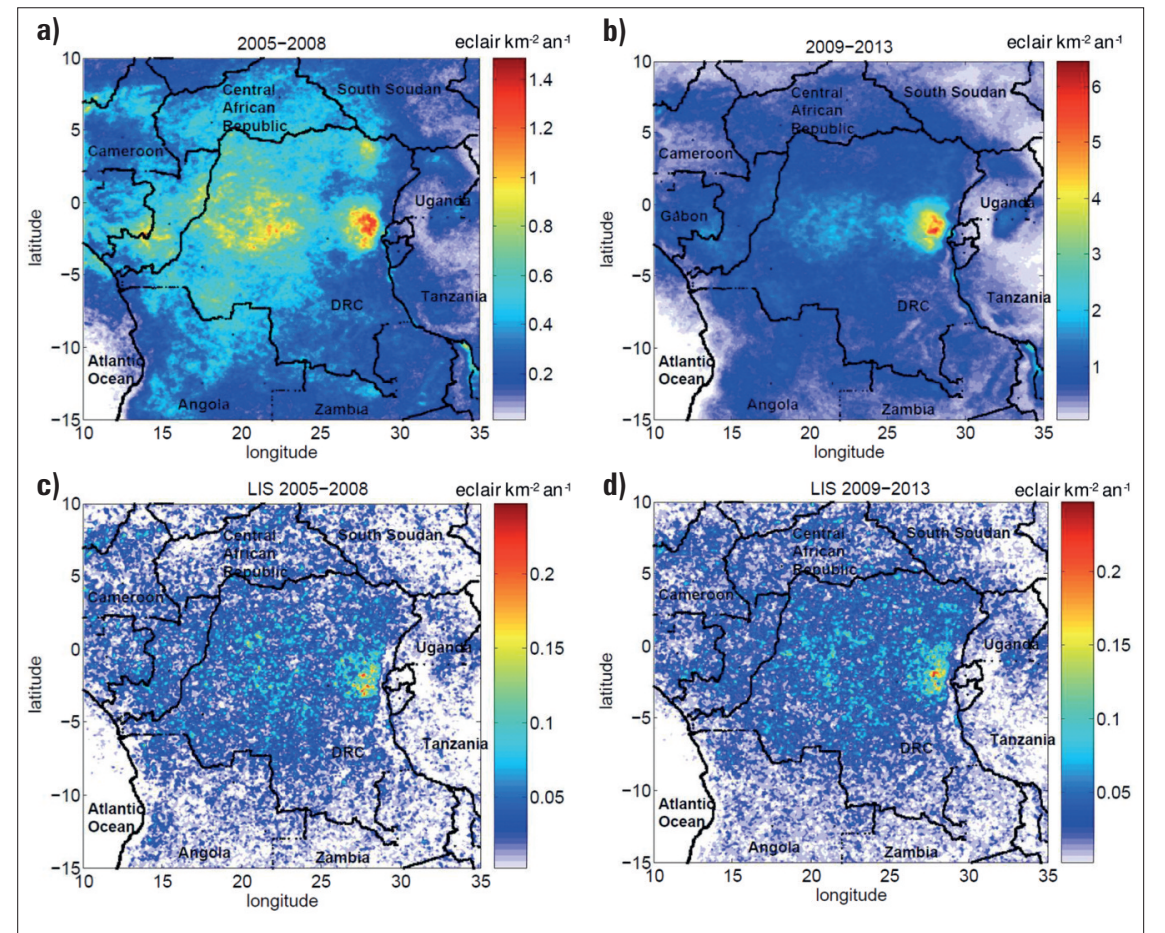

Figure 7. (a) FRD issu du réseau WWLLN pour 2005-2008; (b) pour 2009-2013. (c) FRD issu du capteur LIS pour 2005-2008 ; (d) pour 2009-2013. Tiré de Soula et al. (2016).

\section{Un maximum avec des orages plus actifs ou plus nombreux}

La figure 7 montre une distribution non uniforme de FRD sur la zone. Afin de faire une autre comparaison de l'activité dans les différentes régions, le nombre de jours concernés par les orages est calculé avec une même résolution de $0,1^{\circ} \times 0,1^{\circ}$. La figure 8 montre un exemple de FRD (figure $8 \mathrm{a}$ ) et du nombre de jours d'orages (figure 8 b) pour l'année 2013. Un jour est considéré comme orageux sur un pixel donné si au moins un éclair a été détecté. Tout d'abord, le nombre de jours d'orages est maximal dans la région où FRD l'est aussi. Cela signifie que les valeurs élevées de FRD sont principalement dues au grand nombre de jours d'orages. Cependant, le nombre élevé de jours d'orages est plus étendu. Cela signifie que le maximum 


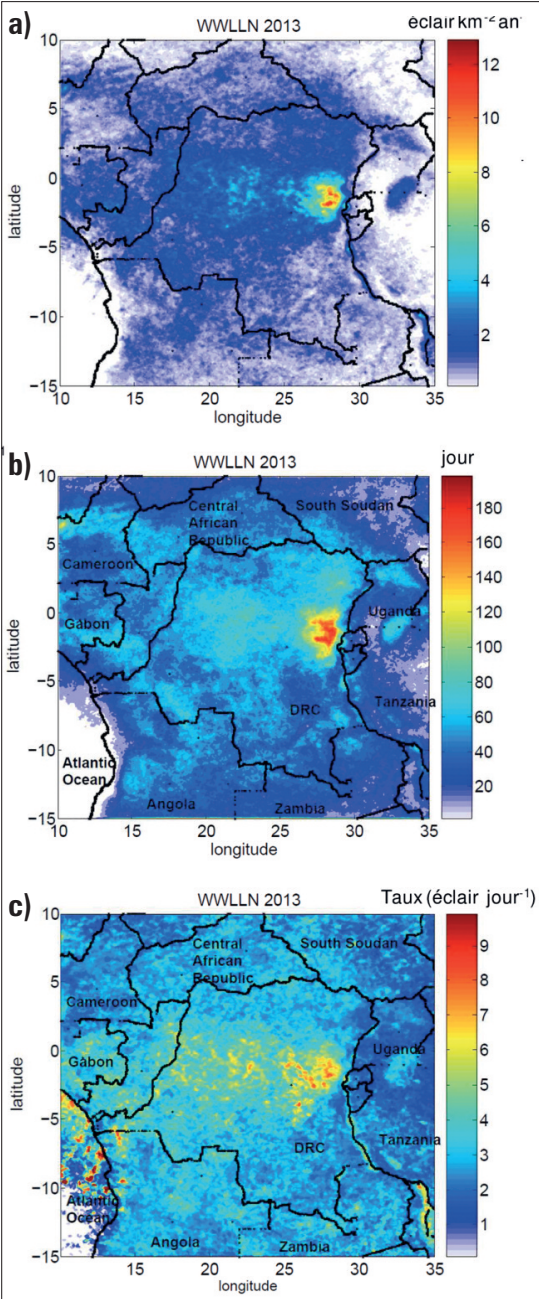

Figure 8. (a) FRD moyen pour 2013 et (b) nombre de jours d'orages pour 2013, d'après les données du réseau WWLLN. (c) Nombre d'éclairs par jour d'orage pour 2013. Tiré de Soula et al. (2016).

de FRD n'est pas seulement dû à un plus grand nombre de jours d'orage, mais également à plus d'éclairs pendant ces jours d'orage. Le nombre moyen d'éclairs par jour d'orage est représenté sur la figure $8 \mathrm{c}$. Ce paramètre est moins contrasté que FRD et plus grand dans les régions d'activité maximale, en particulier dans l'est de la RDC. Dans cette région, le nombre d'éclairs par jour d'orage atteint près de 8 , alors qu'il atteint près de 5 dans la région centrale du bassin du Congo. Cela indique que dans cette région d'activité maximale les orages sont soit plus actifs, soit plus stationnaires, soit plus nombreux, soit plusieurs des trois à la fois. Au cours de la période considérée, la valeur maximale du nombre de jours d'orages est passée de 114 en 2005 à 189 en 2013, tandis que FRD max $_{\text {max }}$ est passé de 1,9 éclair $\mathrm{km}^{-2} \mathrm{an}^{-1}$ en 2005 à 12,9 éclairs $\mathrm{km}^{-2} \mathrm{an}^{-1}$ en 2013 (tableau 1). Comparativement, l'augmentation de FRD est plus forte, ce qui est dû à l'augmentation de l'efficacité de détection du réseau WWLLN.

\section{Un maximum présent en toute saison}

La figure 9 présente la répartition de FRD dans la totalité de la zone et dans la région du maximum, pour trois périodes de 2013 définies en fonction de l'activité annuelle représentée sur la figure 2 : une période de forte activité sur six mois d'octobre à mars, une période de faible activité de trois mois de juin à août et une période intermédiaire de 3 mois comprenant avril, mai et septembre. L'échelle de FRD est la même pour chaque graphe afin de comparer les valeurs des trois périodes. Les trois périodes représentent respectivement 60,3 , 14,6 et $25,1 \%$ du nombre total d'éclairs en 2013, ce qui est proche des proportions moyennes pour l'ensemble de la période de 9 ans (2005-2013), soit respectivement 62,6, 13,8 et $23,6 \%$. Les valeurs maximales de FRD pour les trois périodes sont respectivement de 8,38, 4,33 et 3,67 éclairs $\mathrm{km}^{-2} \mathrm{an}^{-1}$. Les rapports entre ces valeurs maximales de FRD sont inférieurs à ceux entre les nombres d'éclairs, car le maximum de densité est une valeur locale qui n'est pas proportionnelle au nombre total d'éclairs de la zone entière. Pour chaque période, le maximum reste très marqué et dans la même région, mais avec un peu plus de contraste pour la période de forte activité (figure 9a-b). Pour la période de moindre activité orageuse (figure 9c-d), la plupart des éclairs sont situés au nord de l'équateur, sauf dans la région du maximum précédemment décrit et dans une région située à l'ouest concernée par un maximum secondaire. Pendant cette période, l'activité d'éclairs sur les lacs est beaucoup plus faible, c'est-à-dire sur le lac Kivu $\left(29^{\circ} \mathrm{E} ; 2^{\circ} \mathrm{S}\right)$ et le lac Victoria $\left(33^{\circ} \mathrm{E} ; 1^{\circ} \mathrm{S}\right)$.

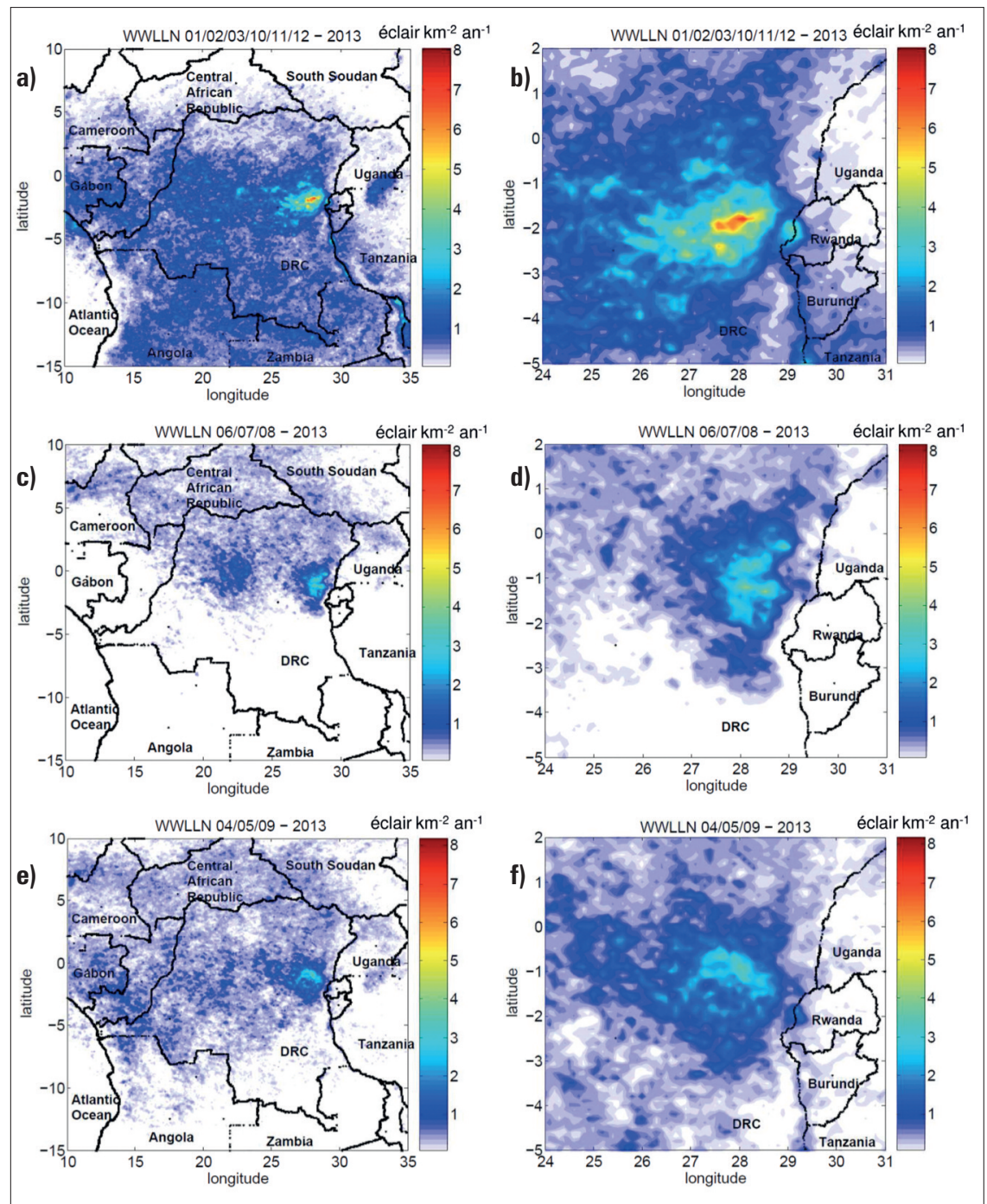

Figure 9. (a)(c)(e) FRD dans la zone d'étude et $(b)(d)(f)$ dans la région du maximum pour trois périodes de 2013 : (a) et (b) janvier-février-mars-octobre-novembre-décembre ; (c) et (d) juin-juilletaoût ; (e) et (f) avril-mai-septembre. Tiré de Soula et al. (201 


\section{Le sud plus exposé que le nord}

Le cycle annuel montre une forte activité entre octobre et mars et une faible activité de juin à août. Même si l'activité orageuse suit le déplacement du soleil au cours de l'année, la répartition des éclairs n'est pas symétrique des deux côtés de l'équateur, alors que la répartition annuelle de l'ensoleillement l'est. En effet, en moyenne sur une période de 9 ans, 56,5\% des éclairs détectés entre $10^{\circ} \mathrm{S}$ et $10^{\circ} \mathrm{N}$ se situent dans 1'hémisphère Sud. Par ailleurs, les plus grandes valeurs de FRD sont principalement situées au sud de l'équateur. La figure 10 montre la distribution méridienne des éclairs détectés par le réseau WWLLN en 2005, 2007 et 2013. Elle confirme la dissymétrie par rapport à l'équateur puisque son maximum est clairement localisé dans l'hémisphère Sud entre $2^{\circ} \mathrm{S}$ et $1^{\circ} \mathrm{S}$ selon les années. Cette dissymétrie au profit de l'hémisphère Sud a également été observée par Waliser et Gautier (1993) pour les nuages à forte réflectivité grâce à l'observation spatiale au-dessus de l'Afrique. En utilisant ce paramètre pour l'emplacement de la zone de convergence intertropicale, ils ont conclu que son emplacement moyen était dans l'hémisphère Sud pendant une durée légèrement plus longue et à des latitudes plus grandes. De même, Žagar et al. (2011) ont trouvé la même dissymétrie et la même forme pour la distribution méridienne des précipitations sur le continent africain en utilisant les données de ré-analyses ERA (de ECMWF-ReAnalysis).

\section{Densité d'éclairs et caractéristiques de surface}

La figure 11 montre une comparaison de FRD moyenné sur toute la période 2005-2013 avec l'orographie, pour toute la zone (figure 11a-b). Le maximum de FRD est d'environ 4 éclairs $\mathrm{km}^{-2} \mathrm{an}^{-1}$ dans l'est de la RDC, soit environ 7 à 8 fois les valeurs moyennes observées dans la plupart des régions du domaine. La plupart des grandes valeurs de FRD sont situées au sud de l'équateur et nous pouvons noter que les valeurs de FRD sont souvent renforcées sur les lacs tels que le lac Victoria $\left(33^{\circ} \mathrm{E} ; 1^{\circ} \mathrm{S}\right)$ ou le lac Tanganyika $\left(34^{\circ} \mathrm{E} ; 10^{\circ} \mathrm{S}\right)$. En revanche, les lacs situés plus au nord

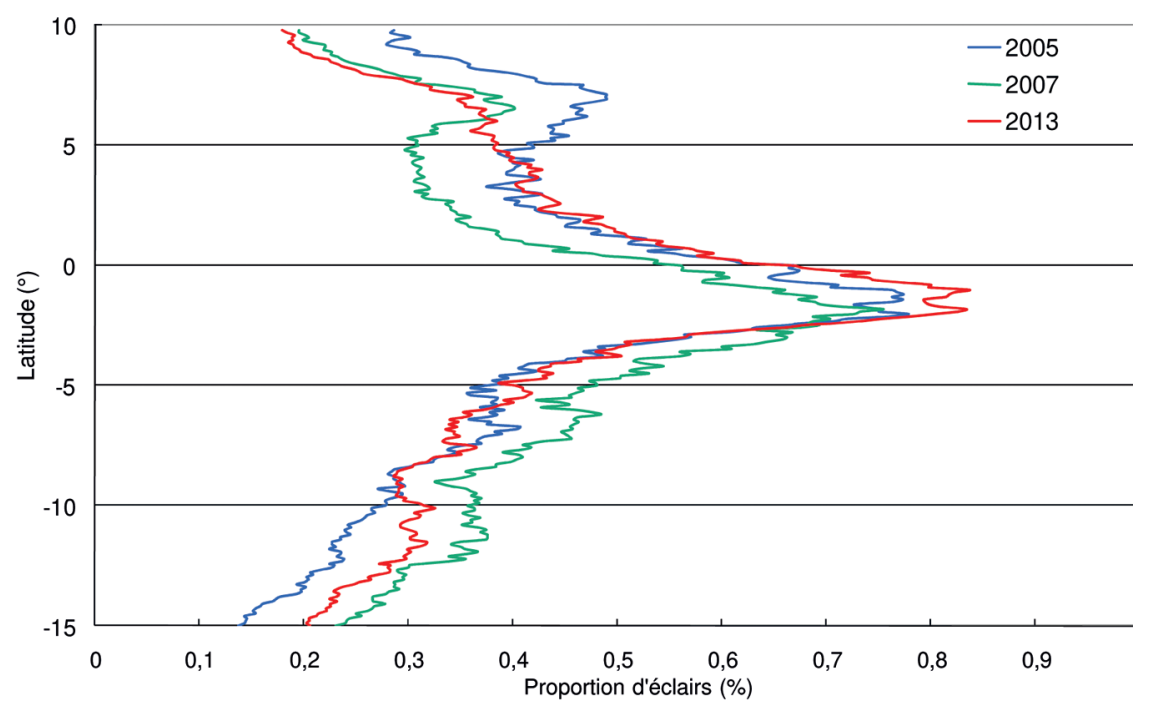

Figure 10. Distribution méridienne des éclairs à partir des données du réseau WWLLN, avec une résolution de 0,1 $1^{\circ}$ pour 2005, 2007 et 2013 dans toute la zone d'étude. Adapté de Soula et al. (2016). présentent apparemment moins de renforcement, comme par exemple le lac Édouard $\left(29,67^{\circ} \mathrm{E} ; 0,67^{\circ} \mathrm{S}\right)$ et le lac Albert $\left(30,67^{\circ} \mathrm{E} ; 1,33^{\circ} \mathrm{N}\right)$. De fortes densités locales sont observées dans la partie occidentale de la région, en particulier à la frontière entre le Congo et le Cameroun $\left(14^{\circ} \mathrm{E} ; 2,5^{\circ} \mathrm{S}\right)$, qui correspond à une augmentation du relief, et sur le littoral gabonais $\left(11^{\circ} \mathrm{E}\right.$; $\left.3,5^{\circ} \mathrm{S}\right)$. Les valeurs moyennes de FRD sur la période 2005-20013 et dans la région du maximum principal sont représentées sur la figure $11 \mathrm{c}$.
L'orographie de la région est présentée sur la figure $11 \mathrm{~d}$. Les lacs Kivu $\left(29,1^{\circ} \mathrm{E} ; 2^{\circ} \mathrm{S}\right)$ et le nord du lac Tanganyika $\left(29,5^{\circ} \mathrm{E} ; 3,5^{\circ} \mathrm{S}\right)$ correspondent à un renforcement de la densité d'éclairs. Par contre, FRD est plus faible au-dessus du lac Édouard $\left(29,7^{\circ} \mathrm{E} ; 0,3^{\circ} \mathrm{S}\right)$ et dans la partie sud du lac Albert $\left(30,8^{\circ} \mathrm{E} ; 1,5^{\circ} \mathrm{N}\right)$. Dans le nord-est de la zone, FRD est plus faible, même au-dessus et autour des plus hautes montagnes de la région à plus de $4000 \mathrm{~m}$ à la frontière entre la RDC et l'Ouganda $\left(29,9^{\circ} \mathrm{E} ; 0,4^{\circ} \mathrm{N}\right)$.

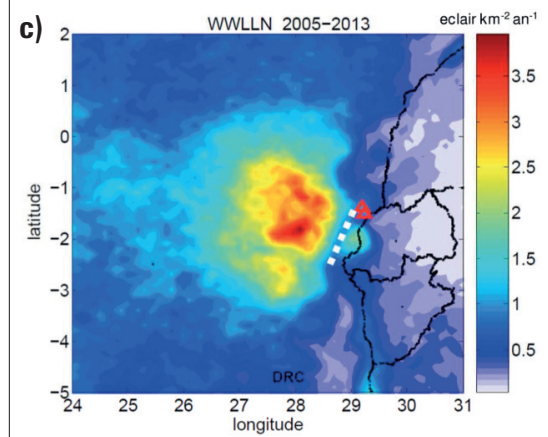

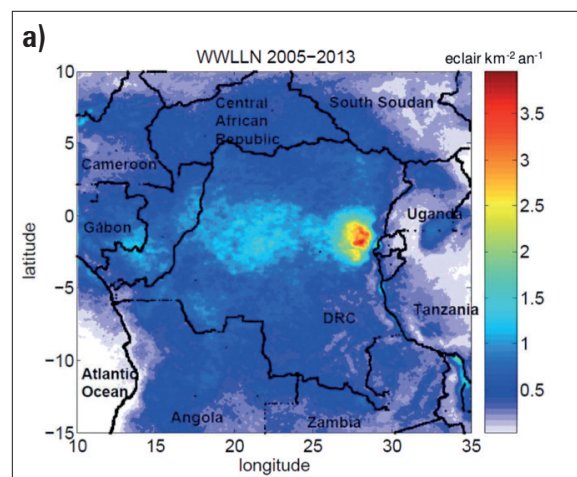

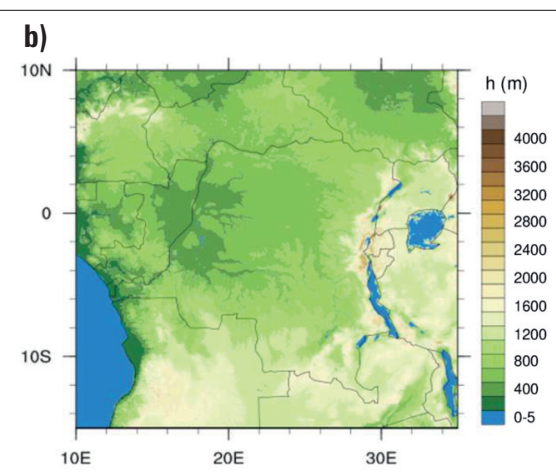

d)

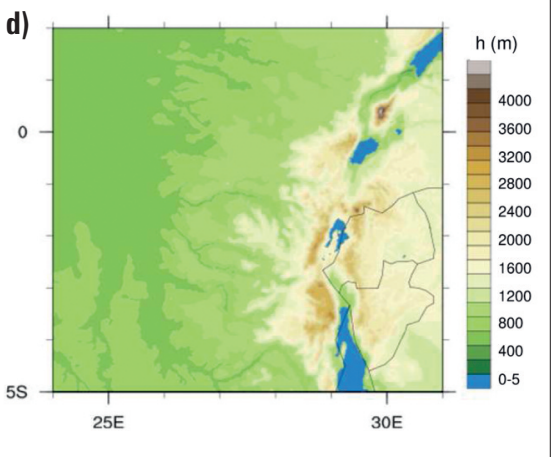

Figure 11. (a) FRD moyen pour la période 2005-2013 dans toute la zone et (c) dans la région du maximum de densité d'éclairs. (b) Altitude (m) dans toute la zone et (d) dans la région du maximum. Adapté de Soula et al. (2016). 
La région avec de fortes valeurs qui a une extension d'environ $300 \mathrm{~km}$ nordsud et $200 \mathrm{~km}$ est-ouest semble présenter deux cœurs de valeurs plus grandes séparés d'environ $75 \mathrm{~km}$, l'un proche de $1^{\circ} \mathrm{S}$ de latitude et l'autre proche de $2^{\circ} \mathrm{S}$. Cette région de fort FRD est située à l'ouest d'une chaîne de montagnes orientée nord-sud caractérisée par des sommets situés à plus de $3000 \mathrm{~m}$ d'altitude près du lac Kivu. Comme les montagnes les plus hautes sont situées à environ $28,5^{\circ} \mathrm{E}$, leur présence et l'orientation de la chaîne (une ligne pointillée blanche sur la figure 11c) peut expliquer le déclenchement de la convection et les grandes valeurs de FRD observées. La présence de lacs à la même latitude, le lac Kivu à petite échelle et le lac Victoria à grande échelle, peut également fournir de l'humidité pour favoriser ou amplifier le développement des nuages d'orage.

\section{Deux régions très actives}

La figure 11a met en évidence deux régions de forte activité, celle du maximum dans l'est de la zone d'étude et l'autre à la même latitude, mais étalée le long de l'équateur. Le maximum de FRD est présent dans la première région tout au long de l'année et peut-être dû à une combinaison de l'arrivée d'air humide de l'est et du soulèvement par le relief situé à l'ouest du lac Kivu. Selon une simulation de la climatologie dans le bassin du lac Victoria réalisée par Anya et al. (2006), les alizés peuvent transporter de l'humidité à grande échelle et augmenter considérablement les précipitations sur le lac. Comme l'explique Ba et Nicholson (1998) qui ont étudié l'activité convective sur les lacs d'Afrique orientale, les précipitations convectives sur le lac Victoria sont à leur maximum tôt le matin. Dans le cas présent, l'humidité peut être transportée depuis le lac Victoria à $500 \mathrm{~km}$ de distance ou l'océan Indien plus à l'est. En effet, selon la climatologie mondiale, peu d'éclairs sont détectés entre l'océan Indien et le maximum est observé dans l'est de la RDC (Christian et al., 2003). La présence de la chaîne de montagnes illustrée sur la figure $11 \mathrm{c}-\mathrm{d}$ est un élément clé du développement des orages susceptibles de produire une forte densité d'éclairs. Une telle situation est aussi observée près du lac
Maracaibo dans l'Etat de Zulia au Venezuela pour laquelle Munoz et al. (2016) montre le rôle d'une topographie complexe et celui du jet nocturne de basse altitude dans le bassin de Maracaibo pour le développement des orages. Pujol et al. (2011) ont utilisé des observations et des simulations pour décrire et quantifier les processus microphysiques impliqués dans le déclenchement, le développement et le renforcement des précipitations convectives par des effets orographiques, en présence d'un lac comme source secondaire d'humidité.

Farnsworth et al. (2011) ont souligné que les systèmes convectifs de moyenne échelle (MCS, pour Mesoscale Convective System) constituaient l'unité fondamentale du transport vertical d'énergie en Afrique centrale. En d'autres termes, la convection dans cette région entraîne généralement la formation de systèmes convectifs. Cette observation est cohérente avec les résultats de Zipser et al. (2006) sur la convection profonde dans le bassin du Congo. Ils ont montré que la convection dans le bassin du Congo dépassait fréquemment la tropopause. La climatologie des MCS en Afrique équatoriale et donc dans tout le bassin du Congo a été donnée par Jackson et al. (2009). À partir d'une série de données de cinq ans, ces auteurs ont montré que la zone entre $5^{\circ} \mathrm{S}$ et $5^{\circ} \mathrm{N}$ qui s'étale de la côte atlantique à l'ouest des hautes montagnes de la vallée du Rift est la plus active en termes d'activité d'orages, car elle inclut deux des quatre maxima du nombre de MCS qu'ils ont identifiés. Dans notre étude, les deux régions très actives coïncident donc avec celles où Jackson et al. (2009) ont trouvé le plus de MCS. De plus, on peut distinguer dans l'étude de Jackson et al. deux cœurs dans la structure de leur région très active : un correspondant à notre maximum et l'autre à une deuxième région assez active plus à l'ouest entre $16^{\circ} \mathrm{E}$ et $25^{\circ} \mathrm{E}$ environ. Les auteurs expliquent l'origine du grand nombre de MCS dans cette vaste région par un maximum de convergence mitroposphérique du côté ouest du courant jet africain oriental de l'hémisphère Sud (AEJ-S). En effet, selon Laing et al. (2008), le cisaillement vertical lié à l'AEJ influence la localisation des systèmes convectifs intenses. De plus, les chaînes de montagnes aident à initier des MCS à longue durée de vie (Laing et al., 2008, 2011). Selon ces auteurs, dans toutes les régions, la convection commence sur le terrain élevé, puis se propage dans des conditions de cisaillement vertical modéré pour se développer en MCS. Par ailleurs et selon plusieurs auteurs, la propagation de la convection en Afrique équatoriale est modulée par les ondes de Kelvin se propageant vers l'est (Laing et al., 2011). Pendant la phase active de ces ondes, les MCS sont plus grands et plus intenses, et pendant leur phase sèche, une convergence en altitude se produit et tend à éliminer la convection profonde et donc la propagation vers l'ouest des MCS. La région du maximum principal combine deux conditions favorables à l'activité des orages : la convergence évoquée par Jackson et al. (2009) et un effet orographique local qui renforce l'effet du premier. La région le long de l'équateur est marquée par une activité soutenue mais plus dispersée à cause de la propagation ou de la régénération vers l'ouest de MCS, à une distance de leur développement initial qui dépend de la phase des ondes de Kelvin (Kigotsi et al., 2018).

\section{Exemple d'un MCS dans la région du maximum}

La figure 12 montre un exemple d'activité orageuse dans la zone du maximum le 25 décembre 2013. Les distributions spatiales de l'activité d'éclairs et de la température de sommet des nuages (CTT, pour Cloud Top Temperature) sont montrées sur la figure 12a et les évolutions temporelles du taux d'éclairs et des caractéristiques nuageuses sur la figure $12 \mathrm{~b}$. À $15 \mathrm{~h} 00$ $\mathrm{TU}$, la couverture nuageuse est déjà importante (environ $60 \times 10^{3} \mathrm{~km}^{2}$ avec des sommets ayant des températures inférieures à $-40{ }^{\circ} \mathrm{C}$ ), mais les sommets ne sont pas encore très froids et le taux d'éclairs est très faible. Après $15 \mathrm{~h} 30 \mathrm{TU}$, le taux d'éclairs augmente sensiblement, des températures plus froides apparaissent progressivement, la taille du système n'évolue pas beaucoup, mais les aires de température froide augmentent. Pendant plusieurs heures, des cellules se développent au sein du système convectif avec un maximum d'activité d'éclairs d'environ 18 et 21 éclairs $\min ^{-1}$ autour respectivement de 19 h 00 et de $21 \mathrm{~h} 30$ TU quand plusieurs cellules sont actives en même temps. Ces taux d'éclairs sont issus du réseau WWLLN 
et sont donc à multiplier d'un facteur 12 environ si on tient compte de l'efficacité de détection dans la zone, qui est autour de $8 \%$ pour l'ensemble des éclairs (intranuage et nuage-sol) en 2013. Les CTT passent par un minimum d'environ $-93{ }^{\circ} \mathrm{C}$ simultanément à ces maximums d'éclairs. Les cellules sont matérialisées sur les images de la figure $12 \mathrm{a}$ par des minimas de CTT et des accumulations d'éclairs. On note donc une forte corrélation entre activité d'éclairs et CTT froide. Pendant son activité jusqu'à environ $2 \mathrm{~h} 00 \mathrm{TU}$ le 26 décembre, le système ne se déplace pas beaucoup, ce qui va entraîner des densités importantes d'éclairs dans la région du maximum de $F R D$, grâce notamment à la régénération de certaines cellules. Ce système constitue un bel exemple de MCS dans la région du maximum d'activité d'éclairs.

\section{Conclusion}

Les caractéristiques spatiales et temporelles de l'activité d'éclairs sont analysées dans le bassin du Congo à partir des données du réseau WWLLN recueillies de 2005 à 2013. Une comparaison avec les données du capteur spatial LIS, qui détecte les éclairs intranuage et nuage-sol dans la zone couverte lors de son passage, montre que, même avec une efficacité de détection très faible, le réseau WWLLN rend bien compte de l'activité orageuse. L'efficacité de détection relative au capteur LIS a été calculée et estimée à 5,9\% en moyenne à la fin de la période, ce qui est compatible avec les valeurs obtenues dans d'autres régions du monde. L'augmentation de l'efficacité de détection au cours de la période explique celle du nombre d'éclairs vus par le réseau WWLLN, de la multiplicité des éclairs et des contrastes dans la distribution de la densité d'éclairs. La distribution annuelle moyenne des éclairs décrit un cycle avec de fortes proportions mensuelles (de 10 à $12 \%$ ) d'octobre à mars et des proportions au moins deux fois plus faibles de juin à août. Nous avons caractérisé un maximum très marqué de l'activité d'éclairs, tant par ses dimensions que par sa forme, sa persistance saisonnière, sa récurrence d'une année à l'autre et son
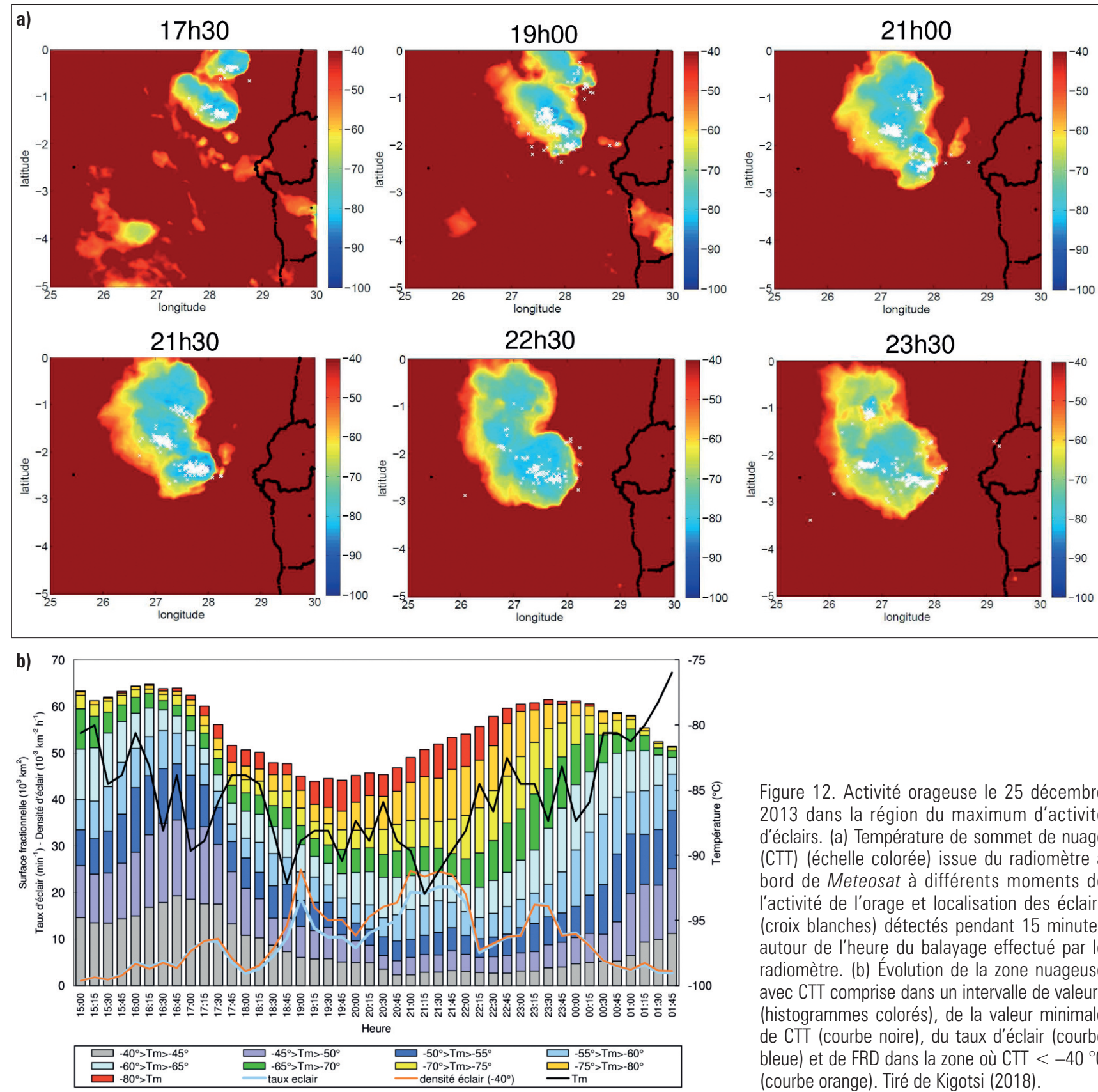

Figure 12. Activité orageuse le 25 décembre 2013 dans la région du maximum d'activité d'éclairs. (a) Température de sommet de nuage (CTT) (échelle colorée) issue du radiomètre à bord de Meteosat à différents moments de l'activité de l'orage et localisation des éclairs (croix blanches) détectés pendant 15 minutes autour de l'heure du balayage effectué par le radiomètre. (b) Évolution de la zone nuageuse avec CTT comprise dans un intervalle de valeurs (histogrammes colorés), de la valeur minimale de CTT (courbe noire), du taux d'éclair (courbe bleue) et de FRD dans la zone où CTT $<-40^{\circ} \mathrm{C}$ (courbe orange). Tiré de Kigotsi (2018). 
environnement géomorphologique et météorologique. L'étude a aussi montré que les orages y sont soit plus nombreux, soit plus intenses, soit plus stationnaires, soit rassemblent plusieurs de ces caractéristiques à la fois. Ce maximum est notamment aligné sur une chaîne de montagne en aval du lac Kivu par rapport aux flux d'air dominants. La distribution méridienne des éclairs a montré un renforcement entre $5^{\circ} \mathrm{S}$ et $5^{\circ} \mathrm{N}$ avec un maximum entre $1^{\circ} \mathrm{S}$ et $2^{\circ} \mathrm{S}$ en partie lié au maximum principal, mais aussi à une forte activité étalée longitudinalement et constituant une zone secondaire de forte activité. L'activité orageuse dans cette zone est variable spatialement d'une saison à l'autre et d'une année à l'autre. La localisation et la forme du maximum principal peuvent s'expliquer par la combinaison de plusieurs facteurs, la présence du relief pour le déclenchement de la convection, un flux d'est et la présence de lacs en amont pour l'alimentation en air humide et le courant jet africain oriental de l'hémisphère Sud (AEJ-S) de basse altitude qui crée les conditions d'instabilité. L'activité plus à l'ouest est nettement moins concentrée, car sa localisation est liée à la distance et à la direction de propagation et de régénération des MCS, en particulier en relation avec la phase des ondes de Kelvin se propageant vers l'est.

\section{Remerciements}

Les auteurs remercient le réseau World Wide Lightning Location Network (www.wwlln.net/) pour avoir fourni les données de localisation des éclairs utilisées dans cette étude. Ils sont reconnaissants à la Nasa et au Global Hydrology Resource Center (GHRC) des États-Unis pour les données du capteur LIS disponibles sur leur site Web.

\section{Bibliographie}

Abarca S.F., Corbosiero K.L., Galarneau T.J., 2010. An evaluation of the Worldwide Lightning Location Network (WWLLN) using the National Lightning Detection Network (NLDN) as ground truth. J. Geophys. Res., 115, D18206.

Albrecht R., Goodman S., Buechler D., Blakeslee R., Christian H., 2016. Where are the lightning hotspots on Earth? Bull. Am. Meteorol. Soc., 97, $2051-2068$.

Anyah R.O., Semazzi F.H.M., Xie L., 2006. Simulated physical mechanisms associated with climate variability over Lake Victoria basin in East Africa. Mon. Weather Rev., 134, 3588-3609.

Ba M.B., Nicholson S.E., 1998. Analysis of convective activity and its relationship to the rainfall over the Rift Valley Lakes of east Africa during $1983-90$ using the Meteosat infrared channel. J. Appl. Meteorol., 37, 1250-1264.

Bovalo C., Barthe C., Begue N., 2012. A lightning climatology of the South-West Indian Ocean. Nat. Hazards Earth Syst. Sci., 12, $2659-2670$.

Cecil D., Buechler D.E., Blakeslee R.J., 2014. Gridded lightning climatology from TRMM-LIS and OTD: Dataset description. Atmos. Res., 135, 404-414.

Christian H.J., Blakeslee R.J., Boccippio D.J., Boeck W.L., Buechler D.E., Driscoll K.T., Goodman S.J., Hall J.M., Koshak W.J., Mach D.M., Stewart M.F., 2003. Global frequency and distribution of lightning as observed from space by the Optical Transient Detector. J. Geophys. Res. 108, 4005. doi: 10.1029/2002JD002347

Dowden R.L., Brundell J.B., Rodger C.J., 2002. VLF lightning location by time of group arrival (TOGA) at multiple sites. J. Atmos. Solar-Terr. Phys., 64, 817-830.

Jackson B., Nicholson S.E., Klotter D., 2009. Mesoscale convective systems over western equatorial Africa and their relationship to large-scale circulation. Mon. Weather Rev., 137, 1272-1294

Kigotsi J., 2018. Analyse de l'activité d'éclairs des systèmes orageux dans le bassin du Congo. Thèse de l'Université Paul Sabatier, Toulouse, France.

Kigotsi J., Soula S., Georgis J.-F., 2018. Comparison of lightning activity in the two most active areas of the Congo Basin. Nat. Hazards Earth Syst. Sci., 18, 479-489.

Laing A.G., Carbone R.E., Levizzani V., Tuttle J.D., 2008. The propagation and diurnal cycles of deep convection in northern tropical Africa. Q. J. R. Meteorol. SoC., 134, 93109.

Laing A.G., Carbone R.E., Levizzani V., 2011. Cycles and propagation of deep convection over Equatorial Africa. Mon. Weather Rev., 129, $2832-2853$.

Orville R.E., Huffines G.R., 2001. Cloud-to-ground lightning in the United States: NLDN results in the first decade, 1989-98. Mon. Weather Rev., 129, 1179-1193.

Pujol 0., Lascaux F., Georgis J.-F., 2011. Kinematics and microphysics of MAP-IOP3 event from radar observations and Meso-NH simulations. Atmos. Res., 101, 124-142.

Rodger C.J., Brundell J.B., Holzworth R.H., Lay E.H., 2008. Growing detection efficiency of the World Wide Lightning Location Network. Am. Inst. Phys. Conf. Proc., 1118.

Soula S., Kigotsi J., Georgis J.-F., Barthe C., 2016. Lightning climatology in the Congo Basin. Atmos. Res., 178-179, 304-319.

Waliser D.E., Gautier C., 1993. A satellite-derived climatology of the ITCZ. J. Clim., 6, 2162-2174.

Žagar N., Skok G., Tribbia J., 2011. Climatology of the ITCZ derived from ERA Interim reanalyses. J. Geophys. Res., 116, D15103. doi:10.1029/2011JD015695.

Zipser E.J., Cecil D.J., Liu C., Nesbitt S.W., Yorty D.P., 2006. Where are the most intense thunderstorms on Earth? Bull. Am. Meteorol. Soc., 87, 1057-1071. 